\title{
Flujo de residuos: Elemento base para la sostenibilidad del aprovechamiento de residuos sólidos municipales
}

\author{
Luis F. Marmolejo *\$, Patricia Torres*, Edgar R. Oviedo*, Diego F. Bedoya*, \\ Claudia P. Amezquita", Rafael Klinger ${ }^{* *}$, Fred Albán ${ }^{* * *}$, Luis F. Diaz \\ * Escuela de Ingeniería de Recursos Naturales y del Ambiente, Universidad del Valle. \\ ** Escuela de Ingeniería Industrial y Estadística, Universidad del Valle. \\ *** Escuela de Ingeniería de Materiales, Universidad del Valle. \\ ****CalRecovery Inc. Estados Unidos \\ §lufermar@univalle.edu.co
}

(Recibido: Septiembre 21 de 2009 - Aceptado: Diciembre 11 de 2009)

\section{Resumen}

En Colombia, la reincorporación de la fracción aprovechable de los residuos sólidos municipales -RSM al ciclo productivo se ha fortalecido con la implementación de Plantas de Manejo de Residuos Sólidos -PMRS. El flujo de residuos permitió establecer interrelaciones entre las características y cantidades de los residuos con las fuentes de generación y las formas de aprovechamiento, obteniendo elementos fundamentales para la sostenibilidad de las PMRS. Mediante su aplicación en un municipio con una PMRS que tipifica las existentes en el país, se determinó que los predios residenciales son la principal fuente de generación de RSM siendo los residuos orgánicos de rápida degradación la categoría generada en mayor proporción; debido a que estos residuos no son separados en la fuente ni acondicionados para su transformación y que las condiciones de operación en la PMRS son inadecuadas, el 70\% termina su ciclo en la disposición final. Los predios residenciales generan las mayores cantidades de materiales reciclables, pero los de origen comercial e institucional son de mejor calidad; el $43.6 \%$ de los reciclables son tomados por recuperadores en el punto de generación, haciendo necesario implementar estrategias de trabajo con los mismos para proyectar el funcionamiento de las PMRS.

Palabras Claves: Aprovechamiento, Flujo de residuos, Manejo integral de residuos, Residuos sólidos municipales.

SANITARY ENGINEERING

\section{Waste flow: a fundamental element for the sustainability of resource recovery from municipal solid wastes}

\begin{abstract}
Resource recovery from Municipal Solid Wastes -MSW has been gained strenght in Colombia with the implementation Solid Waste Management Plants -SWMP. The waste flow allows establishing relations between quantities and characteristics of solid wastes with sources of generation and alternatives for resource recovery and contributes to PMRS sustainability. This study applied the waste flow tool in a typical SWMP of a Colombian municipality and the results show that the residential sector is the main source of MSW and also that the most significant proportion of the solid wastes generated are High Degradation Organic Wastes - HDOW. Seventy percent of HDOW are disposed in landfills because of lack of segregation at source. HDOW neither are conditioned for their transformation and the SWMP do not have a good performance. The highest quantities of recyclable materials were generated in the residential sector; but these materials have lower quality than those generated by commercial and institutional sectors. Forty three of recyclable materials are recovered in their sources by scavengers. To improve the SWMP performance it is important to regulate the interaction between public waste service and scavengers.
\end{abstract}

Keywords: Resource recovery, Solid waste flow, Integrated waste management, Municipal solid waste. 


\section{Introducción}

La relación entre el manejo de los residuos sólidos, la salud y la economía de las comunidades es reconocida mundialmente (Hamer, 2003; Bartone, 1996; Sha'A to et al., 2007), siendo el no generarlos la forma más simple y efectiva de controlarlos (Tonglet et al., 2004). Producto de las estrategias propuestas en eventos de alcance mundial como las Cumbres de Río (1992) y de Johannesburgo (2002), las políticas o jerarquías para la Gestión de los Residuos Sólidos definidas en diferentes países, ubican al aprovechamiento como estrategia base para el manejo de los residuos generados.

En Colombia, en el caso de los residuos sólidos municipales -RSM -, su aplicación formal ha venido materializándose a través de la implementación de Plantas de Manejo de Residuos Sólidos -PMRS cuyo funcionamiento se ha orientado hacia el aprovechamiento y valorización de la mayor cantidad posible de residuos y la disposición final de aquellos materiales que no tienen posibilidad de ser aprovechados o no existen opciones para la transformación o mercadeo en el entorno. El impulso que viene dándose, se refleja en el incremento tanto en el número de PMRS construidas como de municipios atendidos por las mismas, que entre 2006 y 2008, pasó de 34 a 59 y de 68 a 98 respectivamente (SSPD, 2008a). Las PMRS se han construido en mayor grado en poblaciones menores de 20000 habitantes (SSPD 2008a; SSPD, 2008b), las cuales a su vez han sido catalogadas entre los segmentos poblacionales con mayores limitaciones para la gestión de residuos sólidos en el país (Procuraduría, 2003; UNICEF \& Procuraduría, 2006).

Aspectos como la resistencia de las comunidades a la ubicación de sitios de disposición final, la dificultad para conseguir espacios y la reducción de costos de recolección, transporte y disposición final en rellenos sanitarios regionales, han estimulado la implementación de las mismas. Sin embargo, evaluaciones realizadas muestran que en la generalidad de los casos, éstas han tenido deficiencias en su funcionamiento y en varios casos ha sido necesario dejar la infraestructura instalada sin ser utilizada (MAVDT, 2006; SSPD, 2008b).

La sostenibilidad de las PMRS está influenciada por la valorización de los residuos y el posicionamiento de los productos en el mercado, requiriéndose una visión empresarial para el manejo de las mismas. Un elemento básico para alcanzar estos propósitos es el conocimiento y control de las cantidades y características de las materias primas del proceso (residuos sólidos) porque éstas tienen una alta relación con la efectividad de las PMRS y las posibilidades de mercado de los productos, factores que a su vez inciden en su rendimiento económico-financiero y en su sostenibilidad.

Las características de las materias primas a procesar están influenciadas por decisiones o eventos que ocurren en el lugar donde se generan los residuos, en el sitio de presentación, en el vehículo transportador o en la PMRS; el generador es quien decide qué hacer con los residuos, manejándolos como basura (elemento que desecha y en el que no identifica opciones de aprovechamiento) o como residuo (identificándolo como un material que puede ser aprovechado por otros) y tomará decisiones sobre la separación de materiales, el almacenamiento y la presentación de los mismos. De igual forma, el prestador del servicio define aspectos como el tipo y frecuencia de recolección, que también pueden afectar las cantidades y calidad de los materiales. Así mismo, los procesos de separación y transformación realizados en la PMRS incidirán sobre estas variables.

El Reglamento Técnico del Sector Agua Potable y Saneamiento Básico de la República de Colombia - RAS 2000 - (Mindesarrollo, 2000) establece que la caracterización de los residuos debe realizarse al menos una vez cada dos años; sin embargo, esta disposición es poco aplicada posiblemente por razones asociadas con el desconocimiento, la complejidad y costos de las metodologías tradicionalmente aplicadas para la realización de las mismas, lo que guarda relación con lo reportado por Diaz et al. (2002) en cuanto a que en la mayoría de los países en desarrollo se dispone de muy poca información confiable de las 
características de los residuos generados y además que son muy pocos los programas de caracterización de residuos que han sido adecuadamente planeados y ejecutados, corroborando la importancia de establecer metodologías acordes con las condiciones de estas regiones (Hristovski et al., 2007; Klinger et al., 2009).

El análisis del flujo de residuos es una herramienta útil para evaluar la gestión de los materiales o recursos (Binder \& Mosler, 2007), permitiendo además identificar los problemas de contaminación en un sistema (Masó \& Blasi, 2008). El flujo de residuos está ligado tanto a las cantidades y calidad de los materiales como a las prácticas culturales propias del contexto; por tanto, debe complementase con una caracterización (cantidad y composición) que cumpla con criterios de validez y confiabilidad y además con herramientas que exploren las prácticas de manejo y así formular estrategias integrales que optimicen el aprovechamiento de los productos de la PMRS y su posicionamiento en el mercado.

En este artículo se presenta una aplicación de la estimación del flujo de residuos que considera la interrelación entre la caracterización y las prácticas de manejo de residuos sólidos, brindando elementos clave para mejorar la efectividad del aprovechamiento y por tanto la sostenibilidad de las PMRS.

\section{Metodología}

El estudio se desarrolló en la cabecera de un municipio colombiano con una población menor de 20 mil habitantes (9550) que cuenta con PMRS y cuyo servicio de aseo cubre el $100 \%$ de la localidad e incluye la recolección conjunta de los residuos residenciales, comerciales e institucionales de tipo ordinario y el traslado a la PMRS, en donde los materiales se separan manualmente. La conformación y funcionamiento de la PMRS guarda correspondencia con las indicadas por SSPD (2008b) para la generalidad de las PMRS del país. La investigación incluyó las fases de estimación de la cantidad y composición física de los RSM, determinación de prácticas de manejo de RSM y la estimación del flujo de RSM.

\subsection{Estimación de la cantidad y composición física de los RSM}

Para esta estimación se desarrolló un programa de muestreo y caracterización para cada uno de los tres tipos de predio incluidos en el estudio (residencial, comercial e institucional):

En el caso de los predios residenciales, la cantidad generada se estimó a partir de la determinación de la producción percápita (PPC). La determinación del tamaño de muestra se realizó tomando información de la PPC y varianza estimadas en un estudio de caracterización previamente realizado en la localidad (Holguín \& Quintero, 2000). La selección de la muestra se hizo aplicando la técnica del muestreo aleatorio estratificado proporcional (Mendenhall et al., 2006), tomando como referencia la estratificación socioeconómica utilizada por la empresa prestadora de los servicios de agua potable y alcantarillado en la localidad. Para estos predios, simultáneamente a la recolección de las muestras de residuos sólidos, se tomó información sobre el número de personas que las habían generado.

Para los predios comerciales, se efectuó un levantamiento del universo de los mismos y debido a que no habían estudios de caracterización previos, se programó el muestreó aleatorio de al menos el 20\%, obteniéndose respuesta del 14\% de los establecimientos. En el caso de los predios institucionales, se programó censo, pero la respuesta fue del $75 \%$.

En cada uno de los predios seleccionados, se tomaron muestras de los residuos generados durante una semana, las cuales se recogieron y pesaron, previo al paso de los vehículos recolectores del servicio de aseo.

La Tabla 1 resume el universo y número de predios incluidos en el muestreo y caracterización física de los RSM generados en los diferentes tipos de predios. Las categorías de residuos incluidas en la composición física fueron las indicadas en el RAS 2000, incluyéndose además la categoría de residuos higiénicos, la cual hace referencia a residuos generados por la higiene personal. 
Tabla 1. Universo y número de predios incluidos en el estudio

\begin{tabular}{lcc}
\hline Tipo de Predio & Universo & Tamaño de muestra \\
\hline Residencial & 2788 & 53 \\
Comercial & 163 & 23 \\
Institucional & 19 & 11 \\
\hline
\end{tabular}

La información sobre materiales aprovechables con demanda en el mercado regional o local suministrada por los recuperadores informales, centros de acopio y el operador de la PMRS, mostró la necesidad de desagregar las categorías de papel y plástico; el primero fue clasificado en las categorías archivo, plegadiza, periódico, kraft, pulpa moldeada, carbón y aluminio. El plástico fue clasificado tanto por tipo de material (PET, PEAD, PVC, PEBD, PP, PS y otros), como por proceso de transformación (inyección, extrusión, inyección-soplado, termoformado y soplado).

\subsection{Determinación de prácticas de manejo}

En cada uno de los predios incluidos en el muestreo, se aplicó una encuesta que consultaba aspectos relacionados con las actividades económicas desarrolladas, el almacenamiento y presentación de los residuos sólidos y el aprovechamiento interno o la comercialización de residuos. La aplicación de la encuesta se complementó con la observación al interior del predio. En el caso de los predios residenciales se incluyó la caracterización de la composición del grupo familiar y de las actividades económicas desarrolladas al interior de los mismos.

\subsection{Estimación del flujo de residuos}

Se partió de la identificación y análisis de las cadenas de manejo de los residuos y de los actores partícipes en las mismas. Para ello se utilizaron técnicas como talleres con grupos organizados de la comunidad y con el prestador del servicio de aseo, así como la observación directa. De igual forma, se utilizó información obtenida en el muestreo y caracterización física de los residuos y en la encuesta de prácticas de manejo. El flujo se construyó agrupando los materiales en tres categorías:

- Residuos orgánicos de rápida degradación (RSORD): incluye los residuos de comida y de jardín.
- Residuos reciclables: incluye los materiales no incluidos en la categoría de RSORD que tienen potencial de comercialización.

- Materiales no aprovechables o rechazo: aquellos que por sus características físicas, químicas o biológicas no pueden aprovecharse o no tienen demanda en el mercado.

La estimación de las cantidades de residuos generadas en los predios residenciales, comerciales e institucionales se realizó utilizando los resultados de la fase de muestreo y caracterización. La cantidad de residuos que ingresan a la PMRS se estimó a través del pesaje de los materiales que llegan en el vehículo transportador. La cantidad de materiales tomados por los recuperadores informales se determinó estableciendo la diferencia entre los residuos generados en los diferentes predios y la cantidad que llega a la planta. Finalmente, la cantidad de residuos que salen de la PMRS se estimó utilizando los registros de comercialización disponibles en la misma, los cuales están soportados con los respectivos registros de peso y facturas de venta.

\section{Resultados y discusión}

\subsection{Cantidad y composición física de los residuos}

El programa de muestreo realizado mostró una PPC residencial de $0.43 \mathrm{Kg}$ /(hab.día), con un nivel de confianza del $95 \%$ y un error $\delta=12.7 \%$. En estudios previamente realizados en la localidad (Holguín y Quintero, 2001; Alcaldía et al., 2004), se obtuvieron valores de $0.52 \mathrm{Kg} /$ (hab.día) y 0.50 $\mathrm{Kg} /$ (hab.día) respectivamente, mostrando una tendencia al descenso, lo cual puede atribuirse al arraigo de prácticas de aprovechamiento interno (producto de actividades de sensibilización) y al deterioro de la capacidad económica de la población. 
Con la PPC determinada y teniendo en cuenta la población del municipio, la producción total de residuos de los predios residenciales es del orden de $4107 \mathrm{Kg} /$ día. La generación estimada de residuos comerciales fue de $347.8 \mathrm{Kg}$ /día, valor también inferior al reportado en el Plan de Gestión Integral de Residuos Sólidos de la localidad (439 $\mathrm{Kg}$ /día - Alcaldía et al., 2004). En cuanto a los residuos institucionales, se estimó una generación de $133.1 \mathrm{Kg}$ /día.

La producción total de RSM en la localidad es de $4587.9 \mathrm{Kg} /$ día, lo cual indicaría que la PPC de RSM en la localidad es de $0.48 \mathrm{Kg} /$ (hab.día), la cual se aproxima al valor promedio establecido por el RAS-2000 para municipios colombianos de bajo nivel de complejidad (0.45 Kg/(hab.día)) e inferior al indicado por OPS (2005) para poblaciones menores de 15000 habitantes $(0.59$ $\mathrm{Kg} /($ hab.día $)$ ).

En la Tabla 2 se presentan los resultados de la caracterización de la composición física de los residuos; observándose en general el predominio de los RSORD coincidiendo con la tendencia reportada por diferentes fuentes para poblaciones de países en desarrollo (OPS, 2005; UNEP \& CalRecovery, 2005; Trochinetz \& Milhecic, 2009). El valor obtenido en la población objeto de estudio es similar al indicado por MAVDT (2007) para Colombia (65\%). El significativo porcentaje de residuos higiénicos ratifica la importancia de la recomendación hecha por DAPM \& UNIVALLE (2006) en cuanto a la necesidad de incluirlos como categoría independiente en los estándares de clasificación establecidos en las normas

Tabla 2. Composición física de los residuos residenciales en la cabecera del municipio

\begin{tabular}{|c|c|c|c|c|}
\hline Clasificación & Residencial (\%) & Comercial (\%) & Institucional (\%) & Global (\%) \\
\hline Comida & 64.48 & 72.70 & 39.46 & 64.4 \\
\hline Jardín & 5.22 & 1.73 & 42.16 & 6.0 \\
\hline Higiénicos & 8.93 & 3.90 & 1.40 & 8.3 \\
\hline Papel & 3.08 & 7.27 & 7.59 & 3.5 \\
\hline Cartón & 1.24 & 1.06 & 1.20 & 1.2 \\
\hline Plástico & 7.20 & 6.57 & 3.73 & 7.1 \\
\hline Vidrio & 1.15 & 2.55 & 0.89 & 1.2 \\
\hline Metálicos & 1.01 & 0.52 & 0.18 & 0.9 \\
\hline Caucho y cuero & 0.72 & 0.03 & 0.04 & 0.6 \\
\hline Textiles & 2.99 & 1.02 & 1.44 & 2.8 \\
\hline Madera & 0.45 & 0.16 & 0.53 & 0.4 \\
\hline Cerámicos & 1.77 & 0.00 & 0.04 & 1.6 \\
\hline Huesos & 0.38 & 0.01 & 0.01 & 0.3 \\
\hline Otros & 1.36 & 2.46 & 1.32 & 1.4 \\
\hline
\end{tabular}


Tabla 3. Tipos de papel generados con los RSM

\begin{tabular}{lcccc}
\hline Tipo & Residencial (\%) & Institucional (\%) & Comercial (\%) & Total (\%) \\
\hline Archivo & 37.32 & 66.59 & 20.27 & 40.83 \\
Papel plegadiza & 29.95 & 8.40 & 54.98 & 28.15 \\
Papel periódico & 14.94 & 2.54 & 10.68 & 12.74 \\
Papel kraft & 9.05 & 16.97 & 8.84 & 10.27 \\
Pulpa moldeada & 8.74 & 4.20 & 4.23 & 7.75 \\
Papel carbón & 0.00 & 0.89 & 0.27 & 0.16 \\
Papel aluminio & 0.00 & 0.41 & 0.73 & 0.11 \\
\hline
\end{tabular}

Tabla 4. Composición de los plásticos, de acuerdo a su proceso y tipo de materiales asumidos por el estudio, generados en la cabecera municipal.

\begin{tabular}{lccccccc}
\hline $\begin{array}{r}\text { Tipo de } \\
\text { plástico }\end{array}$ & $\begin{array}{c}\text { Inyección } \\
(\%)\end{array}$ & $\begin{array}{c}\text { Inyección- } \\
\text { soplado (\%) }\end{array}$ & $\begin{array}{c}\text { Termo- } \\
\text { formado } \\
(\%)\end{array}$ & $\begin{array}{c}\text { Extrusión } \\
(\%)\end{array}$ & $\begin{array}{c}\text { Soplado } \\
(\%)\end{array}$ & $\begin{array}{c}\text { Coextrusión } \\
(\%)\end{array}$ & Total (\%) \\
\hline PET & 0.00 & 1.19 & 1.08 & 0.00 & 9.12 & 0.00 & 11.39 \\
PEAD & 4.64 & 0.00 & 0.51 & 2.89 & 10.72 & 0.00 & 18.76 \\
PVC & 3.60 & 0.11 & 1.14 & 3.40 & 2.29 & 0.00 & 10.54 \\
PEBD & 2.63 & 0.00 & 1.54 & 23.56 & 0.00 & 0.00 & 27.74 \\
PP & 3.03 & 0.00 & 3.22 & 1.88 & 1.11 & 3.37 & 12.60 \\
PS & 4.03 & 0.00 & 4.80 & 0.02 & 0.00 & 0.00 & 8.86 \\
Otros & 5.08 & 0.00 & 0.33 & 0.03 & 0.36 & 4.31 & 10.11 \\
Total & 23.02 & 1.30 & 12.65 & 31.79 & 23.60 & 7.68 & 100.00 \\
\hline
\end{tabular}

colombianas, así como de almacenarlos y presentarlos separadamente, con el objeto de evitar la contaminación de otros residuos, más cuando se proyecta su aprovechamiento.

La fracción de materiales con potencial de aprovechamiento tales como papel, cartón, plástico, vidrio y metales, es de aproximadamente $14 \%$, valor significativamente inferior al reportado para el país que es de 24\% (MAVDT, 2007). En cuanto a la clasificación del papel, como se muestra en la Tabla 3, en los tres tipos de predios incluidos, se encontró un predominio marcado de las categorías de archivo y plegadiza; el primero tiene una amplia posibilidad de mercado en la zona, mientras que con el segundo ocurre lo contrario ya que tanto el tipo de fibra como el color del material limitan su mercadeo. Actualmente el mercadeo de la plegadiza se ha dificultado por la producción nacional de pulpa mecánica. Dadas estas circunstancias se deben estudiar opciones de mezcla de ambos residuos buscando obtener una fibra con mayor demanda en el mercado.

La Tabla 4 muestra la composición de los plásticos, de acuerdo con su proceso de transformación y tipo de materiales asumidos por el estudio; en cuanto al tipo de resina se observa el marcado predominio de las categorías de polietilenos, PEBD y PEAD, las cuales correspondían principalmente a bolsas, que ameritan lavarse para su aprovechamiento posterior. En cuanto al tipo de proceso de los materiales a aprovechar, es notoria la mayor aplicación de la extrusión, soplado e inyección. 


\subsection{Prácticas de manejo}

El $50 \%$ de las residencias encuestadas no entrega al vehículo recolector al menos un material; esta misma práctica es reportada en $25 \%$ de los establecimientos comerciales y $41.7 \%$ de las instituciones. Esta práctica generalmente se lleva a cabo con propósitos de aprovechamiento interno, venta o entrega a un agente externo. La Figura 1 presenta el porcentaje de viviendas, establecimientos e instituciones que separan total o parcialmente una categoría específica de residuos, observándose un predominio en los residuos de comida, cartón, papel y plástico. Los residuos de comida conservados en el predio generalmente se aprovechan internamente en la alimentación de animales o aplicación en suelos, aunque también se presenta la entrega a terceros.

En el caso del cartón, papel, y plástico, como se observa en la Figura 2, en los predios residenciales que no entregan la totalidad de estos materiales al servicio de aseo, predomina la costumbre de regalarlos; en los comerciales e institucionales predomina la entrega o venta a recuperadores o comercializadores de residuos (Figuras 3 y 4), situaciones que inciden en las cantidades de materiales que llegan a la PMRS.

La Figura 5 muestra el número de recipientes utilizados para almacenar los residuos a entregar al servicio de recolección; como se observa, predomina el uso de un solo recipiente lo cual facilita la mezcla y el deterioro de la calidad de los residuos y a su vez la pérdida de valor económico de los mismos y la reducción de las posibilidades de aprovechamiento. Este resultado también es indicio del desconocimiento o poca valoración de los usuarios por la PMRS. En el caso de categorías de papel como el archivo, se ha encontrado que el precio de un kilogramo de papel mezclado es muy inferior al correspondiente para el material limpio (Kofoworola, 2007; Batool et al., 2008).

Respecto de la presentación de los residuos por parte de los predios residenciales, en el $45.54 \%$ de los casos la tendencia es presentarlos en el momento que pasa el vehículo recolector, mientras que $22.77 \%$ de ellos se anticipa entre 15 y 60 minutos y $28.71 \%$ lo presenta con una anterioridad de una hora o más, lo cual facilita la manipulación de los residuos por parte de recuperadores informales.

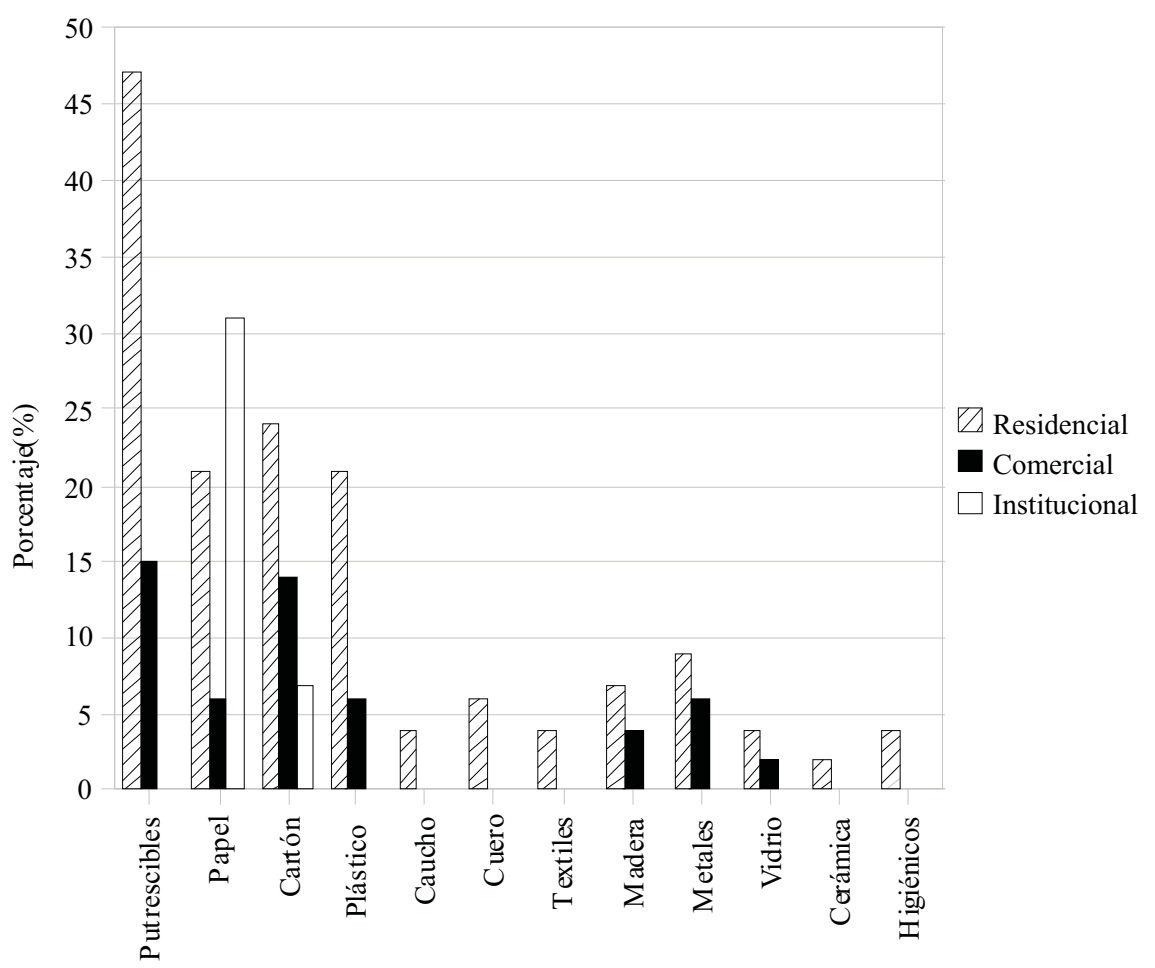

Figura 1. Porcentaje de predios en donde se separa total o parcialmente una categoría específica de residuos 


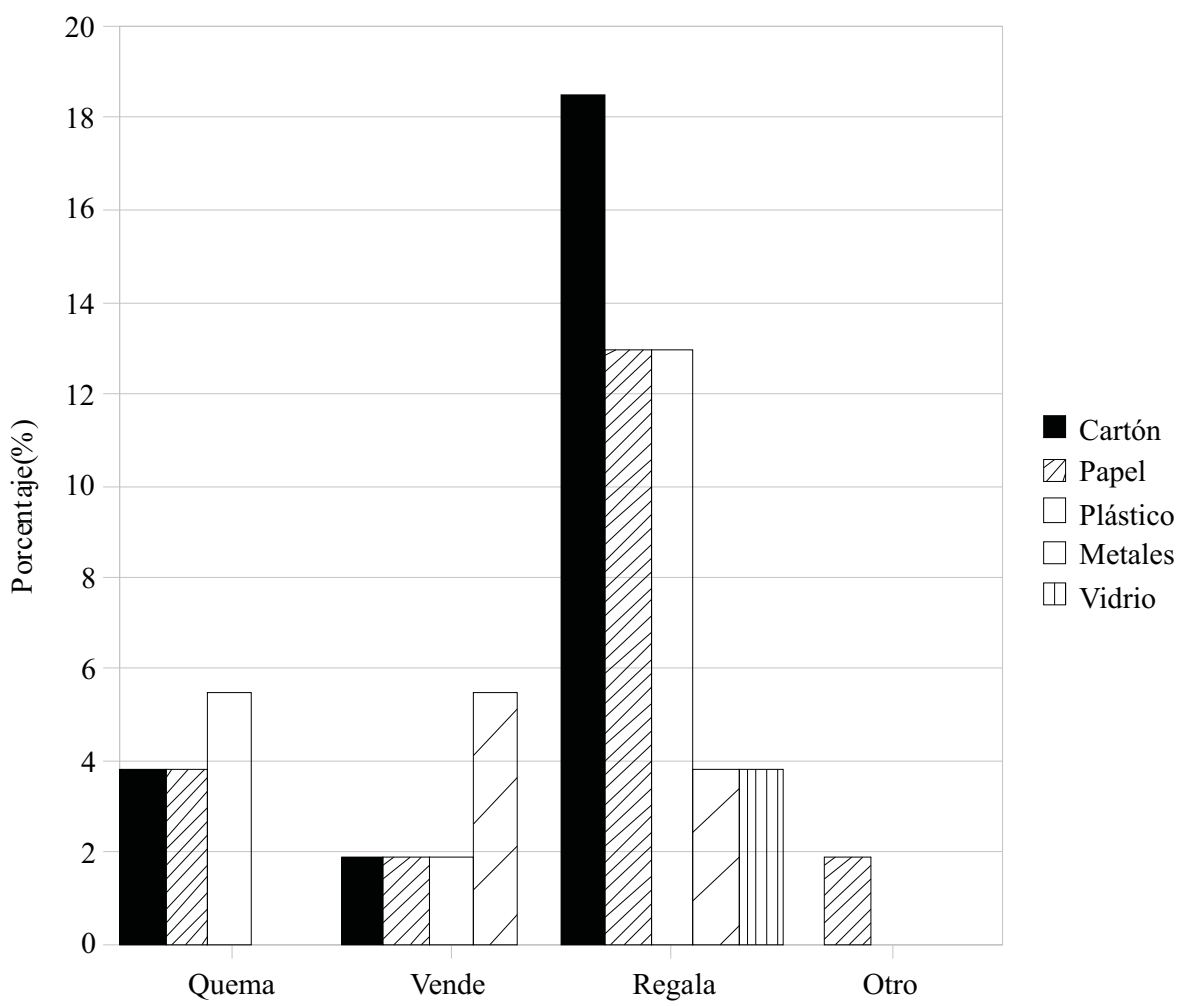

Figura 2. Destino dado a los residuos no entregados al servicio de aseo en los predios residenciales

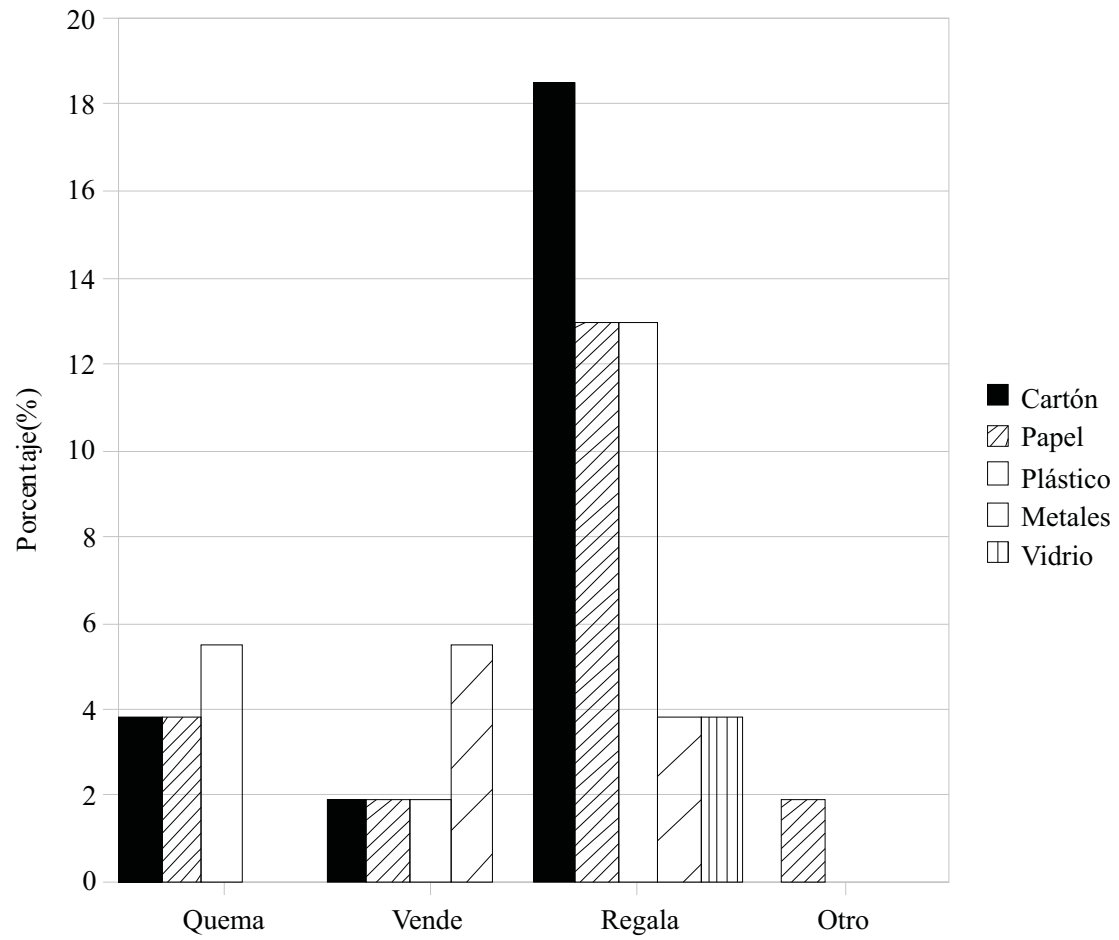

Figura 3. Destino dado a los residuos no entregados al servicio de aseo en los predios comerciales 


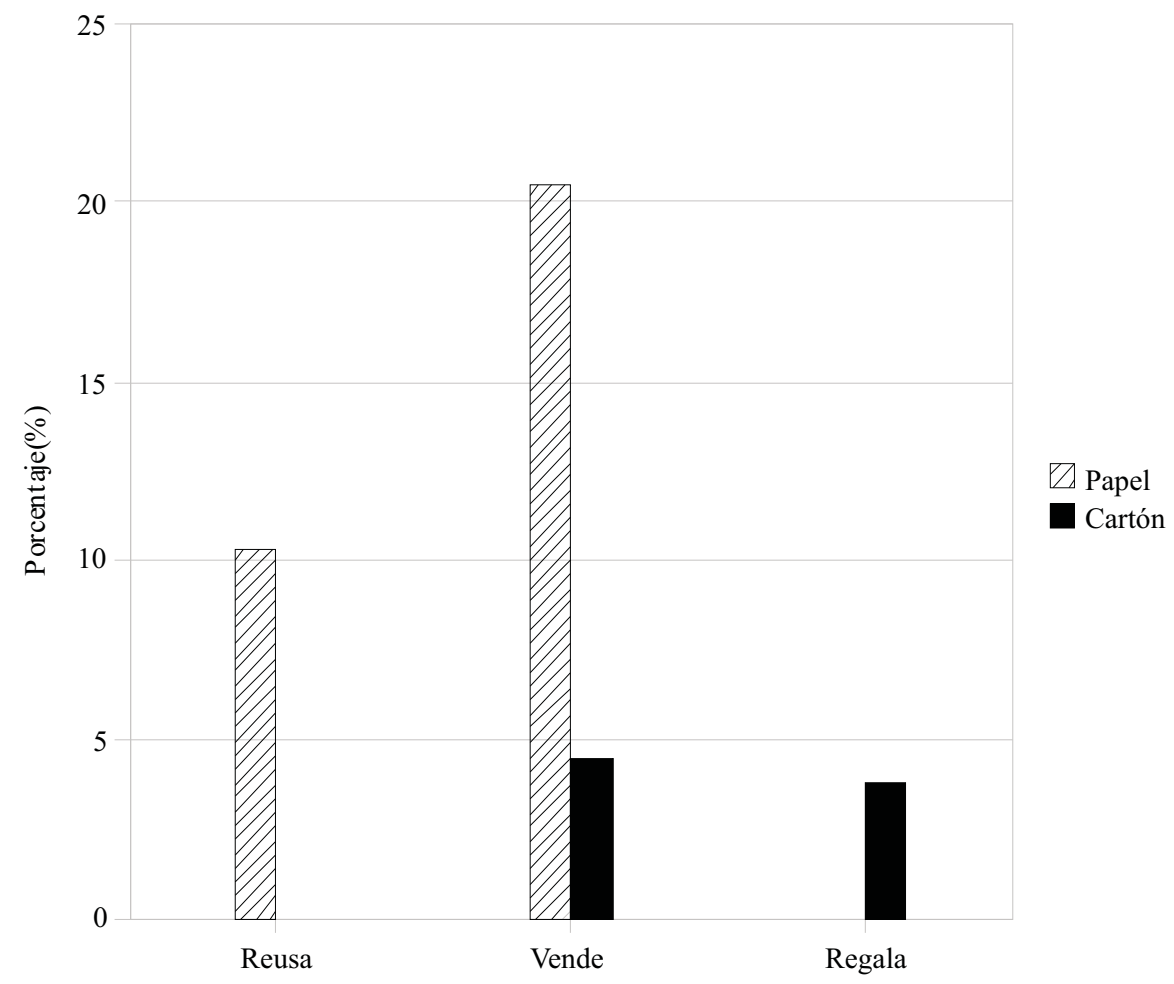

Figura 4. Destino dado a los residuos no entregados al servicio de aseo en los predios institucionales

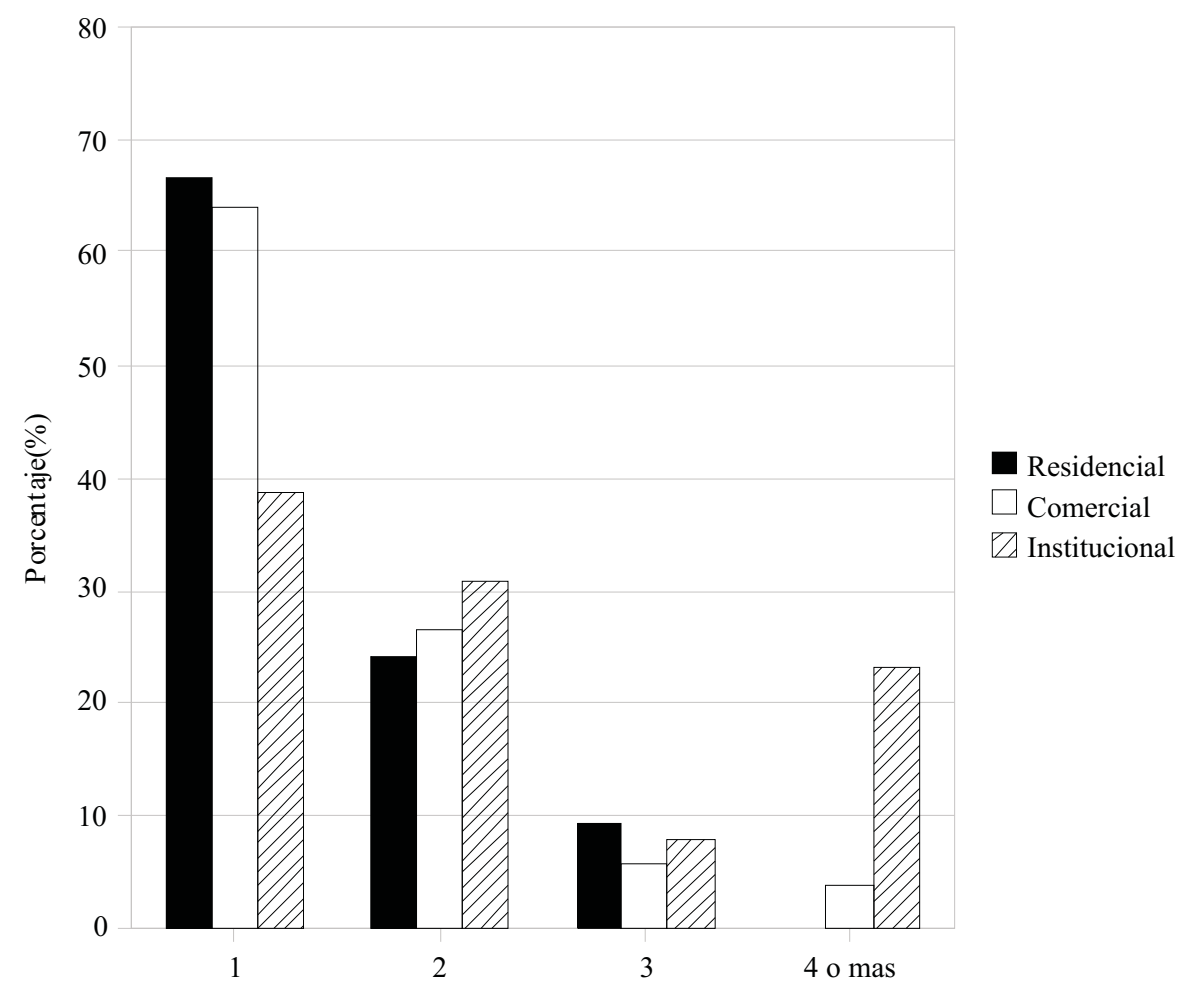

Figura 5. Número de recipientes utilizados para el almacenamiento de los RSM en los predios residenciales, comerciales e institucionales. 


\subsection{Flujo de residuos}

La Figura 6 muestra el flujo de los residuos presentados por los usuarios al servicio de recolección y transporte. En ésta se observan los tres tipos de predios generadores de RSM incluidos en el estudio y las cantidades presentadas al servicio de recolección y transporte y a su vez, las transportadas a la PMRS y las recuperadas por recicladores de la calle. Las cantidades de RSM se distribuyeron según el potencial de aprovechamiento. Esta distribución se realizó de acuerdo con criterios de calidad y mercado en la zona y no implica que hubiera separación en la fuente.

Como se puede deducir de la Figura 6, el 83.5\% de los residuos generados en la localidad, tiene potencial de aprovechamiento. Casi la totalidad de los usuarios presentan los residuos frente al predio, de donde los recuperadores de la calle toman el $5.7 \%$ del total generado (el cual en su totalidad es material reciclable).

La mayor cantidad de RSM presentados, corresponden a la categoría de RSORD; coincidiendo con lo reportado por SSPD (2008b) para el $93.3 \%$ de las PMRS en Colombia, la tecnología proyectada en la localidad para el aprovechamiento de esta fracción de residuos es el compostaje, opción tecnológica con la que se podrían reducir significativamente las necesidades de espacio en sitios de disposición final, disminuyendo la generación de gases y lixiviados y la complejidad del tratamiento de estos últimos (Norbu et al., 2005).

La mezcla de los RSORD con materiales no biodegradables, la poca efectividad de los procesos de separación en la PMRS y las deficiencias en la operación, control y monitoreo del proceso, llevan a que sólo el $30 \%$ de este material sea procesado y que el producto final no se comercialice. Otro elemento a tener en cuenta, es que las mayores cantidades de RSORD provienen del sector residencial, los que usualmente presentan valores en parámetros como el $\mathrm{pH}$ y la humedad que generan dificultades para su tratamiento (Sundberg et al., 2004, Nair et al, 2006). Así mismo, contienen cantidades significativas de residuos de comida preparada, los cuales debido a los hábitos

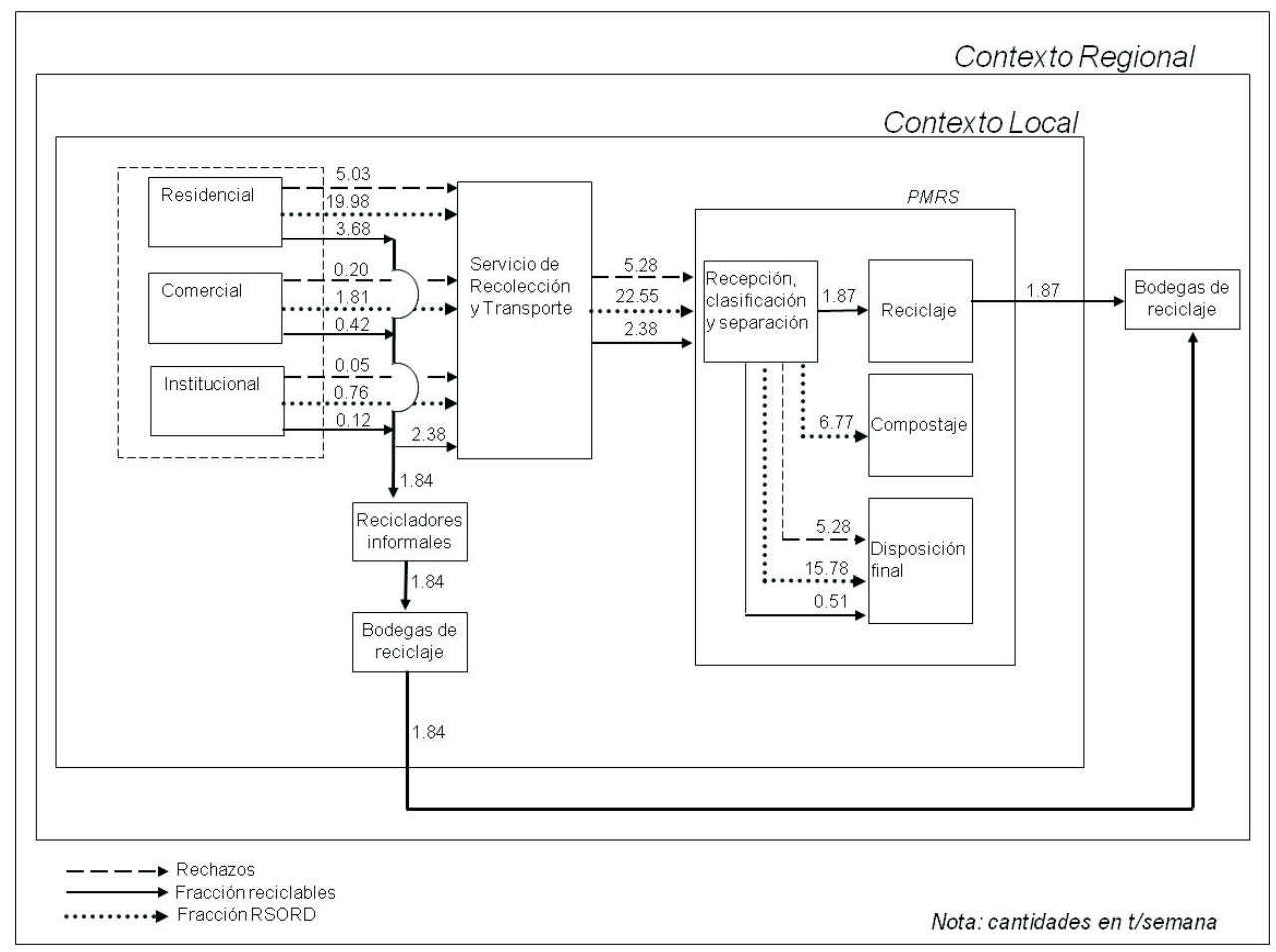

Figura 6. Flujo de los RSM presentados al Servicio de Recolección y Transporte 
alimenticios de la región, tienen contenidos de grasas que hacen más lento el proceso (Chang \& Hsu, 2008) y de sales que pueden limitar el uso del producto final (Dulac, 2001). Tanto en la localidad como en el país, no se reporta la aplicación de procedimientos como el acondicionamiento de la materia prima con materiales de enmienda o soporte que mejorarían el proceso y la calidad de los productos finales (Dulac, 2001, Adhikari et al., 2008).

En el caso del material reciclable, la mayor proporción generada también proviene del sector residencial (87.2\%); sin embargo, las mejores prácticas de almacenamiento y presentación de los residuos en los establecimientos comerciales e institucionales, hacen que los materiales reciclables producidos por éstos sean de mejor calidad. El 43.6\% del material reciclable presentado por los usuarios es tomado por los recuperadores en el sitio de presentación, situación que requiere atención porque además de afectar al usuario o al servicio de recolección (por los derrames de residuos que generalmente se presentan), incide en la sostenibilidad financiera de la PMRS.

Del material reciclable que llega a la PMRS, el $78.6 \%$ es comercializado; para tal efecto y coincidiendo con lo reportado para el $73.3 \%$ de las PMRS del país (SSPD, 2008b), el material es organizado y compactado para disminuir volumen y optimizar el transporte y empacado para almacenarlo y posteriormente venderlo en centros poblados de mayor actividad comercial, ubicados entre 15 y $40 \mathrm{~km}$ de la localidad. Como se observa, el $88.9 \%$ de los residuos reciclables generados en la población se están comercializando a través de los recuperadores de la calle y de la PMRS; esta cifra está por encima del rango reportado por Wilson et al. (2009) para países en desarrollo (20 a $50 \%$ ). En la Tabla 5 se desglosan las cantidades de los materiales reciclables comercializados en la PMRS.

En el caso de materiales como el plástico, cuya comercialización es de $1.25 \mathrm{t} / \mathrm{semana}$ (66.8\% de los materiales comercializados), podrían aplicarse procesos adicionales como la trituración y el lavado, que mejoran la calidad del producto, disminuyendo su volumen, reduciendo los costos de transporte y aumentando su precio de mercado.

El material no aprovechable generado en los predios, que corresponde a $5.28 \mathrm{t} / \mathrm{semana}(16.4 \%$ del total de RSM) incluye materiales como caucho, cuero, textiles, madera, cerámicos, que tienen pocas posibilidades de comercialización en la zona y las categorías de higiénicos, plástico no aprovechable y otros. De otro lado, las condiciones inadecuadas de separación, recolección y proceso en la PMRS hacen que el $60.9 \%$ del material con potencial de aprovechamiento se convierta en rechazo, disminuyendo sustancialmente la vida útil del sitio de disposición final y haciendo más complejo el manejo de éste.

Tabla 5. Cantidades de materiales reciclables comercializados en la PMRS

\begin{tabular}{lc}
\hline \multicolumn{1}{c}{ Materiales } & Kg/semana \\
\hline Plástico Soplado & 187.1 \\
Plástico Inyección & 50.6 \\
PP rígido & 36.6 \\
PET rígido & 80.7 \\
Plástico Película & 891.3 \\
Cartón & 103.8 \\
Chatarra & 170.1 \\
Aluminio & 9.3 \\
Caucho & 16.1 \\
Papel archivo & 83.1 \\
Vidrio & 224.0 \\
Bronce & 1.6 \\
Cobre & 1.2 \\
Hueso & 30.8 \\
\hline
\end{tabular}


El flujo de residuos muestra la necesidad de estimular prácticas de separación en la fuente, recolección selectiva y mejoramiento de los procesos en la PMRS. Adicionalmente, es necesario analizar la relación con los recuperadores de la calle en aras de orientar el funcionamiento de la PMRS y realizar proyecciones financieras; esta relación toma más relevancia en la medida en que se impulsen prácticas como la separación en la fuente y la recolección selectiva, con las cuales la cantidad y calidad de los residuos presentados mejoran y por ende la recuperación en el sitio de presentación resulta más atractiva, lo cual incluso ha estimulado el incremento en el número de recuperadores (Oviedo et al., 2007).

El funcionamiento de la PMRS puede proyectarse con opciones como el solo aprovechamiento de RSORD, estimulando la recolección y recuperación de los reciclables en el sitio de generación por parte de recuperadores organizados. Otra opción, que fue establecida en el municipio colombiano de Guadalupe (Huila) (Municipio de Guadalupe \& UNICEF, 2007), es la implementación de un Acuerdo Municipal, suscrito entre las autoridades locales, el prestador del servicio de aseo y los usuarios en donde el compromiso es hacer entrega de los materiales separados a una organización de recuperadores locales, para que sean aprovechados en la PMRS. Un incentivo para arraigar la separación en la fuente, podría ser que el usuario evidenciara el efecto de estas prácticas en la tarifa del servicio de aseo tanto en el corto como en el mediano y largo plazo. Es importante que el usuario identifique su papel respecto de la sostenibilidad de la PMRS y así además que visualice que beneficios tiene al ser partícipe de dicha sostenibilidad.

\section{Conclusiones}

El flujo de residuos es una herramienta metodológica que brinda información fundamental para la sostenibilidad de las PMRS. Permite precisar las fuentes y tipos de residuos generados, así como el tránsito de los mismos; elementos que tienen relación directa con el funcionamiento de la PMRS y con las proyecciones financieras de las mismas.
Los residuos sólidos orgánicos de rápida degradación - RSORD constituyen los materiales que llegan en mayor cantidad a las PMRS en Colombia y su principal fuente de generación son los predios residenciales. Dado que el compostaje es la opción de aprovechamiento de estos residuos más utilizada en el país, es indispensable la instauración de prácticas que reduzcan su contaminación previa al proceso y adicionalmente su acondicionamiento con materiales de soporte y enmienda. De no acometerse estas prácticas, se afectará la calidad de los productos y por ende su aplicación y mercadeo.

Las actividades de muestreo y caracterización deben articularse con la consulta de las tendencias de las demandas del mercado sobre los distintos tipos de materiales y así verificar la necesidad de desagregación de categorías. Como se observa en la aplicación presentada, la desagregación del papel y del plástico permitió una mejor identificación y estimación de los materiales con potencial de aprovechamiento, así como el planteamiento de opciones para aquellas categorías con menores demandas en el mercado.

Prácticas de manejo como la mezcla de residuos, la entrega de material aprovechable a agentes externos al servicio de aseo y la presentación temprana de los residuos inciden tanto en la cantidad como en la calidad de los productos que llegan a la PMRS. En la medida que a éstas lleguen residuos más limpios, los procesos de separación y acondicionamiento de materiales serán menos complejos y por ende se reducirán los costos de funcionamiento; de otro lado, el valor de mercado de los productos será mayor y así mismo los costos asociados con la disposición final se reducen.

La toma de materiales reciclables por los recuperadores en el lugar de presentación reduce las cantidades de este tipo de residuos que llegan a la PMRS. El estimulo de prácticas como la separación en la fuente y la recolección selectiva, pueden estimular el aumento de estos recuperadores y a su vez disminuir aún más las cantidades; es conveniente que se establezcan estrategias de acuerdo con los recuperadores y con base en ellas realizar las proyecciones de funcionamiento de la PMRS. 


\section{Referencias bibliográficas}

Adhikari, B.K., Barrington S., Martinez, J., \& King S. (2008). Characterization of food waste and bulking agents for composting. Waste Management 28(5), 795-804.

Alcaldía (Alcaldía Municipal de La Victoria), Corporación SUNA HISCA, CVC (Corporación Autónoma Regional del Valle del Cauca). (2004). Plan de Gestión Integral de Residuos Sólidos, Convenio 126-2003. La Victoria-Valle, Republica de Colombia.

Bartone, C.R. (1996). Gestión, recuperación y reciclaje de los desechos municipales: Estrategia para la autosuficiencia en los países en desarrollo. http://www.cepis.ops-oms.org/acrobat/gestion.pdf

Batool, S.A., Chaudhry, N., \& Majeed, K. (2008). Economic potential of recycling business in Lahore, Pakistan. Waste Management 28 (2), 294-298.

Binder, C.R., \& Mosler. H.J. (2007). Wasteresource flows of short-lived goods in households of Santiago de Cuba. Conservation and Recycling $51(2), 265-283$.

Chang, J., \& Hsu, T. (2008). Effects of compositions on food waste composting. Bioresource Technology 99 (17), 8068-8074.

DAPM (Departamento Administrativo de Planeación Municipal) \& UNIVALLE (Universidad del Valle) (2006). Caracterización de los residuos sólidos residenciales generados en el municipio de Santiago de Cali. Informe final del Proyecto Diagnóstico de la composición y caracterización de los residuos sólidos residenciales generados en el municipio de Santiago de Cali. Colombia.

Diaz, L.F., Eggerth, L.L., Savage, G.M. \& Golueke, C.G. (2002). The role of composting in the management of solid wastes in economically developing countries. In Appropriate Environmental and solid waste management and technologies for Developing Countries, Vol 2. ISWA. Istanbul, Turkey. P. 1353-1361.
Dulac, N. (2001). The Organic Waste flow in Integrated Sustainable Waste Management- In Integrated Sustainable Waste Management. A set of five tools for decision makers. Experiences from the Urban Waste Expertise Programme (1995-2001). Series editor Scheinberg, A. Waste. The Netherlands.

Hamer, G. (2003). Solid waste treatment and disposal: effects on public health and environment safety. Biotechnology Advances 22 (1-2), 71-79.

Holguín, J.E., \& Quintero, P.A. (2001). Plan para el Manejo Integral de los Residuos Sólidos generados en el municipio de La Victoria, Valle del Cauca. Proyecto de grado. Programa de Ingeniería Sanitaria. Universidad del Valle, Cali, Colombia.

Hristovski, K., Olson, L., Hild, N., Peterson, D., \& Burge, S. (2007). The municipal solid waste system and solid waste characterization at the municipality of Veles, Macedonia. Waste Management 27 (11), 1680-1689.

Klinger, R.A., Olaya, J., Marmolejo, L., \& Madera, C. (2009). Plan de muestreo para la cuantificación de residuos sólidos residenciales generados en las zonas urbanas de ciudades de tamaño intermedio. Revista Facultad de Ingeniería Universidad de Antioquia 48, 76-86.

Kofoworola, O.F. (2007). Recovery and recycling practices in municipal solid waste management in Lagos, Nigeria. Waste Management 27 (9), 11391143.

Masó, A.M., \& Blasi, A.B. (2008). Evaluation of composting as a strategy for managing organic wastes from a municipal market in Nicaragua. Bioresource Technology 99 (11), 5120-5124.

MAVDT (Ministerio de Ambiente, Vivienda y Desarrollo Territorial). (2006). Política nacional para la gestión integral de residuos sólidos en Colombia. En: VI Congreso Internacional Disposición Final de Residuos Sólidos y Perspectivas Ambientales. Pereira, República de Colombia. 
MAVDT (Ministerio de Ambiente, Vivienda y Desarrollo Territorial). (2007). Evaluación de las cadenas de reciclaje. Bogotá D.C. República de Colombia.

Mendenhall, III., Scheaffer, W., \& Ott, R. (2006). Elementos de muestreo, España: Thomsom.

Mindesarrollo (Ministerio de Desarrollo Económico). (2000). Reglamento Técnico del Sector Agua Potable y Saneamiento Básico -RAS 2000. Título F - Sistemas de Aseo Urbano. República de Colombia.

Municipio de Guadalupe \& UNICEF (Fondo de las Naciones Unidas para la Infancia - Colombia) (2007). Participación Comunitaria en la construcción de políticas públicas municipales. En: Ponencias Seminario Internacional Gestión Integral del Residuos Sólidos. Latinosam 2007. Conferencia Latinoamericana de Saneamiento. Santiago de Cali, Colombia, Noviembre de 2007.

Nair, J., Sekiozoic, V., \& Anda, M. (2006). Effect of pre-composting on vermicomposting of kitchen waste. Bioresource Technology 97 (16), 20912095.

Norbu, T., Visvanathan, C., \& Basnayake, B. (2005). Pretreatment of municipal solid waste prior to landfilling. Waste Management 25(10), 9971003.

OPS (Organización Panamericana de la Salud) (2005). Informe de la Evaluación Regional de los Servicios de Manejo de Residuos Sólidos en América Latina y el Caribe. Washington. D.C.

Oviedo, R., Rodríguez, C., Navarro, L., Quintero, P., \& Marmolejo, L. (2007). Avances en la implementación de la gestión integral de los residuos sólidos en el municipio de La VictoriaValle del Cauca. Avances en investigación y desarrollo en Agua y Saneamiento para el cumplimiento de las metas del milenio (Capítulo VIII). En (Restrepo, I., Sánchez, L.D., Galvis, A., Rojas, J., Sanabria, I.J.). Programa editorial de la Universidad del Valle, Cali, Colombia.
Procuraduría (Procuraduría General de la Nación Delegada para Asuntos Ambientales y Agrarios) (2003). Informe de Seguimiento de Gestión de los residuos sólidos en Colombia. Republica de Colombia.

Sha'Ato, R., Aboho S.Y., Okentude, F.O., Eneji, I.S., Unazi, G., \& Agwa, S. (2007). Survey of solid waste generation and composition in rapidly growing urban area in Central Nigeria. Waste Management 27 (3), 352-358.

SSPD (Superintendencia de Servicios Públicos Domiciliarios). (2008a). Situación de la disposición final de residuos sólidos en Colombia. http://www.superservicios.gov.co/siteSSPD/docu mentos/documentos_pub/48_3682.pdf.

SSPD (Superintendencia de Servicios Públicos Domiciliarios) (2008b). Diagnóstico sectorial plantas de aprovechamiento de residuos sólidos. Republica de Colombia.

Sundberg, C., Smårs, S, \& Jönsson, H. (2004). Low $\mathrm{pH}$ as an inhibiting factor in the transition from mesophilic to thermophilic phase in composting. Bioresource Technology 95 (2), 145-150.

Tonglet, M., Phillips, P.S., \& Bates, M.P. (2004). Determining the drivers for householder proenvironmental behaviour: Waste minimization compared to recycling. Resources, Conservation \& Recycling 42 (1), 27-48.

Troschinetz, M., \& Milhecic, J.R. (2009). Sustainable recycling of municipal solid waste in developing countries. Waste Management 29 (2), 915-923.

UNEP - United Nations Environment Programme \& CalRecovery Incorporated (2005). Solid Waste Management, Vol. 1. United Nations Environment Programme. 
UNICEF (Fondo de las Naciones Unidas para la Infancia - Colombia), \& Procuraduria (Procuraduría General de la Nación). (2006). La infancia, el agua y el saneamiento básico en los Planes de Desarrollo Departamentales y municipales. Bogotá. Republica de Colombia.

Wilson, D., Araba, A.O, Chinwah,K., \& Cheeseman C.R. (2009). Building recycling rates through the informal sector. Waste Management 29 (2), 629-635. 